\title{
An Experimental Analysis of the Effect of Reflections on the Performance of Visible Light Positioning Systems in Warehouses
}

\author{
Yousef Almadani*, Muhammad Ijaz ${ }^{*}$, Sander Bastiaens ${ }^{\dagger}$, Sujan Rajbhandari ${ }^{\ddagger}$, Wout Joseph $^{\dagger}$, and David Plets ${ }^{\dagger}$ \\ * Manchester Metropolitan University, Department of Engineering, Manchester, United Kingdom \\ $\dagger$ Ghent University/imec, Department of Information Technology, Ghent, Belgium \\ $\ddagger$ University of Coventry, School of Computing, Electronics and Mathematics, Coventry, United Kingdom \\ m.ijaz@mmu.ac.uk, sujan.rajbhandari@ coventry.ac.uk, david.plets@ugent.be
}

\begin{abstract}
In this paper, an experimental evaluation of the effect of multipath reflections on two 3D positioning algorithms is experimentally compared. The VLP algorithms use the received signal strength (RSS) for estimating the receiver's 3D position without prior knowledge of its height. An experimental overall median accuracy of $10.5 \mathrm{~cm}$ was achieved in a $4 \times 4 \times 4.1$ $\mathrm{m}^{3}$ test area. The effect of multipath reflections was recreated using a storage shelf rack that is similar to the ones used in warehouses and was placed $26 \mathrm{~cm}$ away from the path. The results demonstrate the degrading effect of reflections on two and three-dimensional positioning systems. The reflections effect was especially severe due to reflections from a metal beam. The achieved median error using the two different algorithms were 7.5 and $6.6 \mathrm{~cm}$ before the addition of a shelf rack, and it increased to 11.7 and $12 \mathrm{~cm}$ after the shelf rack was added. Multipath reflections increased the median positioning errors by $112 \%$ in 2D systems and by $69 \%$ for $3 \mathrm{D}$ systems. The paper demonstrates the degrading effect of multipath reflections on VLP systems and highlights the need to take it into consideration when evaluating VLP systems.
\end{abstract}

Index Terms-visible light positioning, indoor positioning, indoor localization, localisation, visible light communication, multipath, reflections, industrial, warehouses

\section{INTRODUCTION}

The latest advancements and research in visible light communication (VLC) technology using existing lighting infrastructure for communication have paved the way and provided a great opportunity for indoor localization as the infrastructure is already widely available. Visible light positioning (VLP) systems have been analyzed extensively in the literature and demonstrated the potential for high-accuracy tracking, the technology is additionally advantageous due to its immunity from electromagnetic interference.

However, the majority of VLP indoor localization systems are developed for a two-dimensional (2D) positioning system that calculates only the $(x, y)$ coordinates. In order to have a three-dimensional (3D) positioning system, the height of the receiver must be known. Nevertheless, some of the literature presented a 3D positioning system yet assumed the height of the receiver to be known in advance. Some actual 3D positioning system has been presented that would require the use of additional sensors such as an altimeter.
Furthermore, the presence of multipath reflection is a major factor in the performance degradation of VLP systems and has not been widely discussed. Researchers in [1] analyzed the effect of reflections and found that the multipath reflections considerably decrease the positioning accuracy especially around the edges and corners of the room. The work in [2] studied the performance of eight different cost metrics for a VLP system under the presence of reflections and reported median errors between 6.7 and $8.7 \mathrm{~cm}$. The researchers have also found a nearly linear increase in positioning error when the wall reflectance coefficient is increased. The work in [3] examined the performance of VLP systems in three actual office environments. The proposed VLP method locates the receiver via trilateration/multi-lateration if over three light sources are perceived along with an optimization process or uses a fusion method with an inertial measurement unit if less than three signals are received. The achieved $90^{\text {th }}$ percentile positioning errors for the three environments are $0.4 \mathrm{~m}, 0.7$ $\mathrm{m}$, and $0.8 \mathrm{~m}$. An accuracy of $1.1 \mathrm{~m}$ was achieved when only a single transmitter is available. The severity of multipath reflections in industrial environments has been demonstrated in [4] for a flexible manufacturing cell. When comparing the channel impulse response between residential and industrial environments, it is evident that VLC systems an industrial environments suffer greatly from the presence of metallic objects. The use of orthogonal frequency division multiplexing (OFDM) is generally proposed in VLC system as a way to mitigate the effect of multipath reflections. The work in [5] proposed the use of OFDM in a VLP system and reported simulation results with a root mean square (RMS) error of $0.04 \mathrm{~m}$ when OFDM is used while it is $0.43 \mathrm{~m}$ for when onoff keying (OOK) modulation is used. The method, however, is yet to be verified experimentally.

In this paper, we experimentally assess the performance of two different 3D VLP positioning algorithms in a realistic industrial scenario with the presence of a storage rack to examine the effect of multipath reflections on the positioning accuracy. The two positioning algorithms considered in this paper are the Cayley-Menger determinant (CMD) the linearleast square (LLS) [6]. The two algorithms build on a cost 


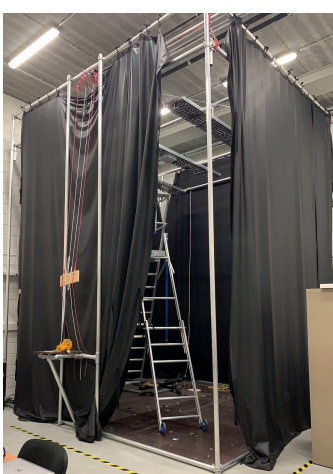

(a)

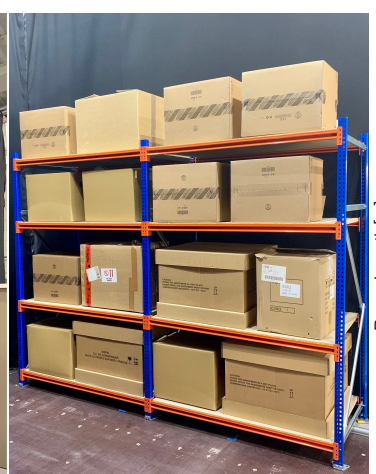

(b)

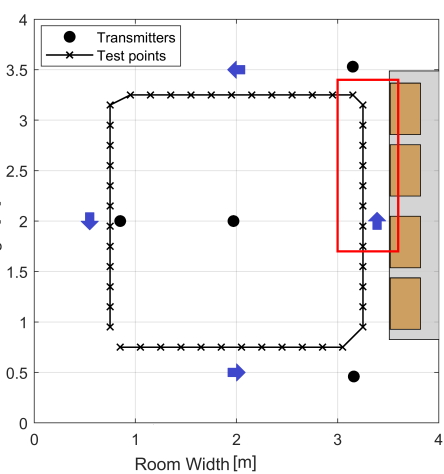

(c)

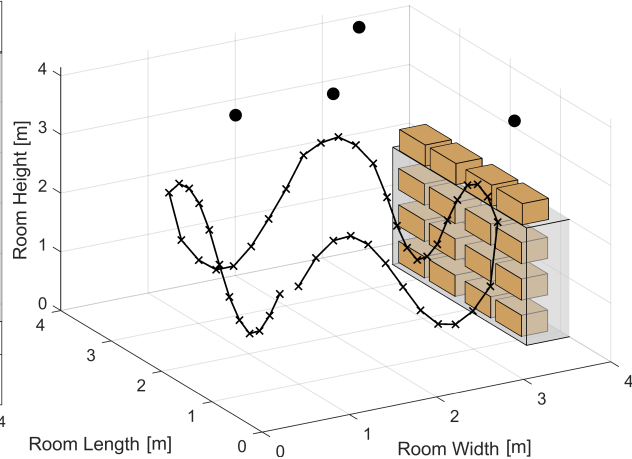

(d)

Fig. 1: (a) The VLP test lab; (b) the shelf rack used in the experiment; (c) the test path demonstrated inside the VLP lab; (d) a $3 \mathrm{D}$ view of of the path.

function to estimate a true $3 \mathrm{D}$ positioning without prior knowledge of the receiver's height.

The remainder of the paper is organized as follows. Section II presents the experimental setup and the system model. Results and discussions are presented in Section III. Conclusions are finally drawn in Section IV.

\section{Materials And Methods}

\section{A. Experimental Setup}

The proposed 3D algorithm is analyzed experimentally in a VLP lab that measures $4 \mathrm{~m} \times 4 \mathrm{~m}$ with the height of the LEDs at approximately $4.1 \mathrm{~m}$, as shown in Fig. 1 (a). Black curtains are used as a substitute for walls to ensure that uncontrolled reflections from walls and objects are avoided. Four BXRE50C3001-D-24 LEDs are intensity-modulated and transmitting pulse trains with a duty cycle of 0.5 with frequencies of $500 \mathrm{~Hz}, 1 \mathrm{kHz}, 2 \mathrm{kHz}$, and $4 \mathrm{kHz}$. This ensures that the contributions from the different LEDs can be demultiplexed individually at the receiver's side.

The receiver is a commercial photodiode with an integrated electrical amplifier (PDA36A2 by Thorlabs) with an active area $A_{p d}$ of $13 \mathrm{~mm}^{2}$ and a responsivity of $0.22 \mathrm{~A} / \mathrm{W}$. The receiver is attached to a tripod with a vertical pole that allows the user to vary the receiver's height. The data is acquired using National Instrument's USB-6212 for processing and a fast Fourier transform (FFT)-based demodulation is used to extract the received power values from each LED in MATLAB $^{\circledR}$ as specified in [7].

Fig. 1 (c) shows a top-view path consisting of fortyeight points selected to take the receiver around the room at different heights ranging from 0.64 to $2.55 \mathrm{~m}$ as shown in Fig. 1 (d). Twenty-five power value readings were collected at each location to reduce the impact of noise. The LEDs are placed in a 'star' configuration with a central LED circularly surrounded by the other three LEDs. This is performed due to an ambiguity issue when the LEDs are placed in a square shape as reported in [6], [8]. Therefore, a star-shaped configuration was proposed in order to encounter this. The experiments has been performed with and without the inclusion of the shelf rack shown in Fig. 1 (b). The height of the shelf rack is $2 \mathrm{~m}$ and has a length of $2.66 \mathrm{~m}$. It has a height of $2.36 \mathrm{~m}$ when stocked with boxes and is placed $26 \mathrm{~cm}$ away from the path. The cardboard boxes have a reflectivity that ranges between $33-42 \%$ depending on the color shade.

\section{B. System Model}

The radiation of an LED chip follows a Lambertian radiation pattern and by only considering the line-of-sight $(\mathrm{LoS})$ path between the LED transmitters and the receiver, the received power is modeled as:

$$
P_{r i}=P_{t i} \frac{(m+1) A_{p d}}{2 \pi d_{i}^{2}} \cos ^{m}(\alpha) \cos (\beta) T_{p d}(\beta) G_{p d}(\beta)
$$

where $P_{t i}$ is the transmitted power from the $i^{t h}$ LED, $m$ is the Lambertian order, $d_{i}$ is the distance between the $i^{t h}$ LED transmitter and the receiver, $\alpha$ is the angle of irradiance, and $\beta$ is the angle of incidence. The optical filter's gain $T_{p d}(\beta)$, and the optical concentrator's gain $G_{p d}(\beta)$ are assumed to be equal to 1 . Additionally, by assuming that the transmitters and the receiver are horizontally parallel, $\cos (\alpha)=\cos (\beta)=$ $\frac{h_{L E D}-z}{d_{i}}=\frac{h}{d_{i}}$, then the $d_{i}$ can be estimated as $\widehat{d}_{i}$ using the received signal power:

$$
\widehat{d}_{i}=\sqrt[m+3]{\frac{(m+1) A_{p d} P_{t i} h^{m+1}}{2 \pi P_{r i}}}
$$

where $h=h_{L E D}-z$ is the unknown vertical height difference between the $\operatorname{LED}_{i}$ transmitter and the receiver $(x, y, z)$. Since $h$ is an unknown, the estimated distance $\widehat{d}_{i}$ cannot be directly calculated from $P_{r i}$ without knowing $h$, or equivalently, $z$. Due to this, a set of estimated distances $\widehat{d}_{i}$ is generated for different receiver heights, $z$, ranging from a minimum height $h_{\min }$ to maximum height, $h_{\max } \leq h_{L E D}$ with $1 \mathrm{~mm}$ intervals.

The actual power of the LEDs can vary from their advertised values by up to $20 \%$ as investigated in [9]. Therefore, a calibration step is performed for each transmitter by collecting one measurement directly under each transmitter $(\alpha=\beta=0)$. 
TABLE I: Summary of the system parameters

\begin{tabular}{cc}
\hline Parameter & Value \\
\hline Room Width x Length x Height & $4 \mathrm{~m} \times 4 \mathrm{~m} \times( \pm) 4.1 \mathrm{~m}$ \\
Transmitters' Power - $P_{t}$ & $13.3 \mathrm{~W}-16.6 \mathrm{~W}-16.4 \mathrm{~W}-16.1 \mathrm{~W}$ \\
Transmitter's semi-angle & $60^{\circ}$ \\
Receiver's Height Range $-z$ & $0.64-2.55 \mathrm{~m}$ \\
Photodetector's Area - $A_{p d}$ & $13 \mathrm{~mm}^{2}$ \\
Receiver's Responsivity & $0.22 \mathrm{~A} / \mathrm{W}$ \\
\hline
\end{tabular}

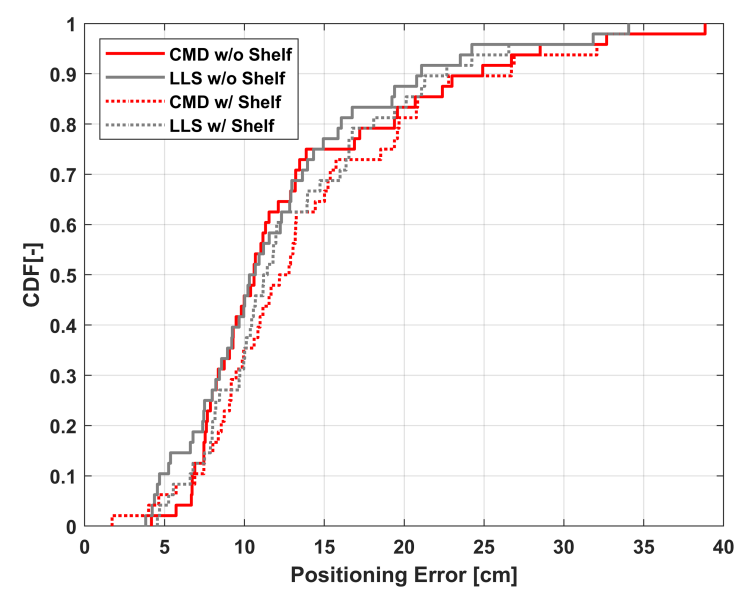

Fig. 2: CDF of the errors with and without the shelf rack

Then the estimated transmitted power is calculated using $P_{t i}=\frac{P_{r i} 2 \pi d_{i}^{2}}{A_{p d}(m+1)}$ [8]. Table. I lists the transmitted power for each transmitter.

As mentioned previously, two different trilateration algorithms are used to estimate the position of the receiver. The two algorithms are the Cayley-Menger determinants [10] and the linear least square method [6]. Because the unknown height of the receiver is needed for a $3 \mathrm{D}$ position estimation, the two algorithms estimate positions for a range of possible PD heights ranging from the ground up to the height of the LEDs. Once all of the possible receiver locations have been generated using both algorithms, the final and most probable 3D position of the receiver is found at the minimum of the cost function $C(h)$ as [6]:

$$
\begin{aligned}
C(h)= & \frac{1}{N} \sum_{i=1}^{N}\left[\widehat{d}_{i}(h)\right. \\
& \left.-\sqrt{\left(\widehat{x}(h)-x_{i}\right)^{2}+\left(\widehat{y}(h)-y_{i}\right)^{2}+(h)^{2}}\right]^{2}
\end{aligned}
$$

where $C(h)$ is the average squared error between the estimated distances $\widehat{d}_{i}$ using (2), and the distances of the estimated 3D location of the unknown receiver $(\widehat{x}, \widehat{y}, \widehat{z})$.

\section{RESULTS}

The first test was performed along the specified path shown Fig. 1 (c) and (d) without the inclusion of the shelf rack. Fig. 2 demonstrates the cumulative distribution function (CDF) of the errors performed along the specified path, the 3D positioning accuracy reported a median of $10.6 \mathrm{~cm}$ using
TABLE II: A Summary of the median errors for the nine highlighted points.

\begin{tabular}{ccccc}
\hline Positioning Error [cm] & 2D LLS & 2D CMD & 3D LLS & 3D CMD \\
\hline Star without shelf & 5.4 & 4.8 & 7.5 & 6.6 \\
Star with shelf & 12.6 & 9.1 & 11.7 & 12 \\
Percentage increase & $133 \%$ & $90 \%$ & $56 \%$ & $82 \%$ \\
\hline
\end{tabular}

the LLS algorithm and $10.5 \mathrm{~cm}$ using the CMD algorithm. While the reported maximal values $\left(90^{\text {th }}\right.$ percentile) were $20.4 \mathrm{~cm}$ using the LLS algorithm and 23.5 using the CMD algorithm. The performance of both algorithms is comparable in this instance. The test was repeated after the shelf rack was added and reported a median error of 12.5 and $11.3 \mathrm{~cm}$ using the LLS and the CMD algorithm, respectively. The maximal positioning error was $27 \mathrm{~cm}$ when the LLS algorithm was used and $24 \mathrm{~cm}$ using the CMD algorithms, as shown in Fig. 2. Representing median increases of $18 \%$ using the LLS algorithm and $8 \%$ using the CMD algorithm when compared to the positioning errors obtained without the shelf rack.

Given that the shelf rack only aligns the path on one side, a closer inspection of that area would give a better understanding of the effect of multipath reflections in VLP systems. The area that is highlighted in red in Fig. 1 (c) and that is closest to the shelf rack is further examined. The area consists of nine test points with heights ranging from 0.74 to $2.15 \mathrm{~m}$ (The shelf rack measures $2.36 \mathrm{~m}$ when stocked with boxes). Which is why the immediate test points preceding and following the closely examined area with heights greater than $2.36 \mathrm{~m}$ are not be examined as they are higher than the shelf rack. A top-view of the estimated 2D and 3D positions is shown in Fig. 3 (a) without the shelf rack and in Fig. 3 (b) after the shelf rack has been added. A clear degradation in performance of the algorithms can be observed. The performance of the algorithms for 2D and 3D positioning is displayed in Fig. 3 in order to show that reflections affect the performances of $2 \mathrm{D}$ and $3 \mathrm{D}$ positioning systems along the $(x, y, z)$ axis.

For these nine points, the median $2 \mathrm{D}$ error achieved using LLS without a shelf rack reported a median of $5.4 \mathrm{~cm}$. When using CMD, the achieved median error was $4.8 \mathrm{~cm}$. The median errors in a 3D system increased slightly to $7.5 \mathrm{~cm}$ (a median increase of 39\% from 2D) using LLS and $6.6 \mathrm{~cm}$ using CMD (an increase of 38\% from 2D). Table. II lists the median errors and it can be clearly seen that the impact of reflections nearly doubles the median error.

Fig. 4 shows the CDF of the 3D errors for the nine points in a 3D positioning system with and without the shelf rack. After the inclusion of the shelf rack, the reported 3D median error using the LLS algorithm was $11.7 \mathrm{~cm}$ and it was 12 $\mathrm{cm}$ when the CMD algorithm was used. This translates to an median increase of $56 \%$ for the 3D LLS algorithm and an increase of $82 \%$ for the 3D CMD algorithm when compared to the median error before the shelf rack was added. Without the addition of the shelf rack, the lowest 3D positioning error achieved was $4.2 \mathrm{~cm}$ using the LLS algorithm and the highest is $13.5 \mathrm{~cm}$. Using the CMD algorithm had a minimum error of 


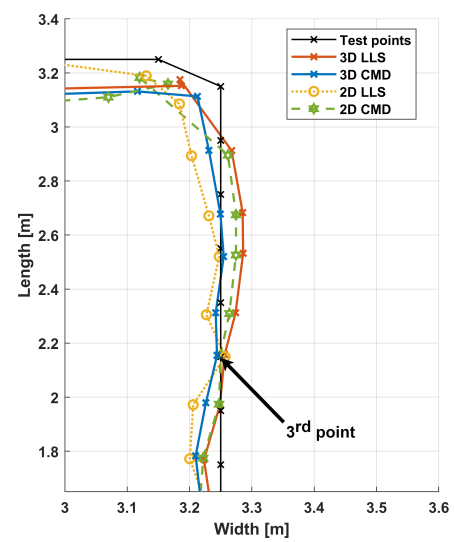

(a)

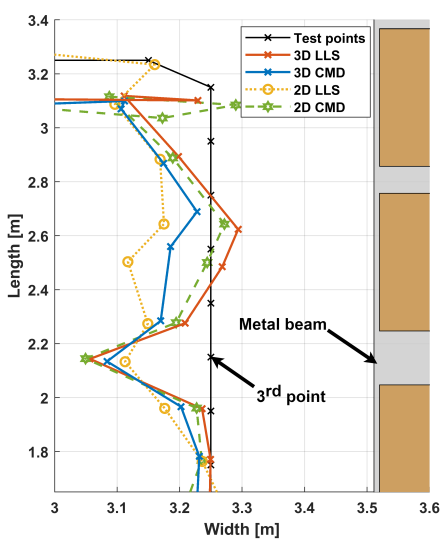

(b)
Fig. 3: The estimated 2D and 3D path for the examined area; (a) without the shelf rack; (b) with the shelf rack.

$4.2 \mathrm{~cm}$ and the highest reported error was $12.9 \mathrm{~cm}$. After the inclusion of a shelf rack, the highest 3D reported error using the LLS algorithm increased to $19.7 \mathrm{~cm}$ for the point directly opposite the metal beam (see Fig. 3 (b)) and the maximum reported error using CMD is $26.6 \mathrm{~cm}$ for the same location. For this specific point ( $3^{\text {rd }}$ from the bottom as shown Fig. 3), the positioning error using the LLS algorithm increased by 13 $\mathrm{cm}$ and by $18 \mathrm{~cm}$ using the CMD algorithm. Reflections are predominant in this specific location due to the location of the middle LED which causes large reflections as this impinges perpendicularly on the beam to the receiver's position. A similar effect can be observed at the points on top (points 7-9), which can be attributed to reflections from the top and bottom horizontal beams as well (the ones in orange in Fig. 1 (b)).

It is worth noting that some of the errors could be attributed to several other factors such as the LED radiation pattern not being perfectly Lambertian or the receiver and LEDs having small unknown tilt angles.

These results, and especially for the point opposite a metallic object, highlight the damaging effects of multipath reflection in VLP systems, an area that is often overlooked in the literature with the assumption of only LOS signals are received.

\section{CONCLUSION}

In this paper, the effect of multipath reflections on two 3D positioning algorithms were compared via experiments. The effect of reflections was recreated using a storage shelf rack that is similar to the ones used in storage facilities. The results show a median increase in terms of positioning errors by $112 \%$ in $2 \mathrm{D}$ systems and by $69 \%$ for 3D systems. The multipath reflections from a metal beam especially worsened the performance of the VLP system. The results also show that the LLS algorithm performs better for the points closest to the shelf rack, while the CMD algorithm achieves a better accuracy overall. The paper demonstrates the degrading effect

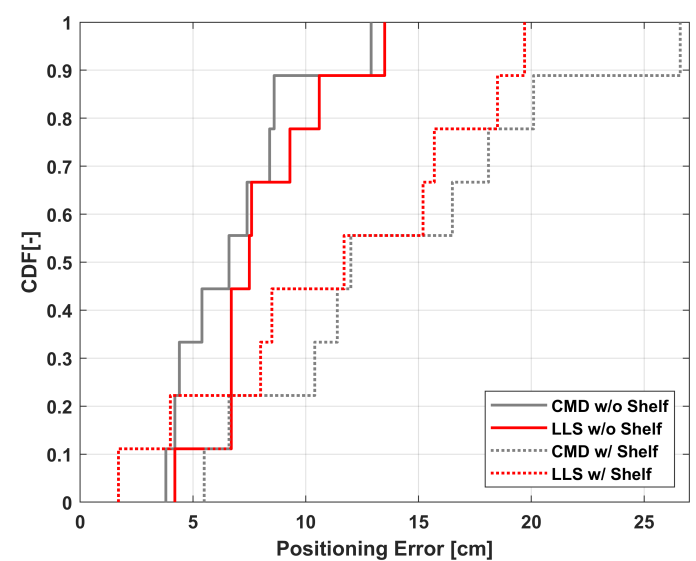

Fig. 4: $\mathrm{CDF}$ of the 3D errors for the nine highlighted points with and without the shelf rack

of multipath reflections on VLP systems and highlights the need to take it into consideration when evaluating a VLP system. Future work consists of analyzing the performance of the VLP system in the presence of a variety of reflective surfaces.

\section{REFERENCES}

[1] W. Gu, M. Aminikashani, P. Deng, and M. Kavehrad, "Impact of multipath reflections on the performance of indoor visible light positioning systems," Journal of Lightwave Technology, vol. 34, no. 10, pp. 25782587, 2016.

[2] D. Plets, A. Eryildirim, S. Bastiaens, N. Stevens, L. Martens, and W. Joseph, "A performance comparison of different cost functions for rss-based visible light positioning under the presence of reflections," in Proceedings of the 4th ACM Workshop on Visible Light Communication Systems, ser. VLCS '17. New York, NY, USA: ACM, 2017, pp. 37-41. [Online]. Available: http://doi.acm.org/10.1145/3129881.3129888

[3] L. Li, P. Hu, C. Peng, G. Shen, and F. Zhao, "Epsilon: A visible light based positioning system," in 11th USENIX Symposium on Networked Systems Design and Implementation (NSDI 14). Seattle, WA: USENIX Association, 2014, pp. 331-343.

[4] M. Uysal, F. Miramirkhani, O. Narmanlioglu, T. Baykas, and E. Panayirci, "Ieee 802.15.7r1 reference channel models for visible light communications," IEEE Communications Magazine, vol. 55, no. 1, pp. 212-217, January 2017.

[5] M. Aminikashani, W. Gu, and M. Kavehrad, "Indoor positioning with ofdm visible light communications," in 2016 13th IEEE Annual Consumer Communications Networking Conference (CCNC), Jan 2016, pp. 505-510.

[6] D. Plets, Y. Almadani, S. Bastiaens, M. Ijaz, L. Martens, and W. Joseph, "Efficient 3d trilateration algorithm for visible light positioning," Journal of Optics, vol. 21, no. 5, p. 05LT01, apr 2019.

[7] S. De Lausnay, L. De Strycker, J. Goemaere, N. Stevens, and B. Nauwelaers, "A visible light positioning system using frequency division multiple access with square waves," in 2015 9th International Conference on Signal Processing and Communication Systems (ICSPCS), Dec 2015, pp. 1-7.

[8] D. Plets, S. Bastiaens, M. Ijaz, Y. Almadani, L. Martens, and W. Joseph, "Three-dimensional visible light positioning: an experimental assessment of the importance of the leds' locations," in 2019 International conference on Indoor Positioning and Indoor Navigation (IPIN), Pisa, Italy, 2019, accepted.

[9] D. Plets, S. Bastiaens, L. Martens, W. Joseph, and N. Stevens, "On the impact of led power uncertainty on the accuracy of $2 \mathrm{~d}$ and $3 \mathrm{~d}$ visible light positioning," Optik, vol. 195, p. 163027, 2019.

[10] F. Thomas and L. Ros, "Revisiting trilateration for robot localization," IEEE Transactions on Robotics, vol. 21, no. 1, pp. 93-101, Feb 2005. 